\title{
Symbolic Encoding of Periodic Orbits and Chaos in the Rucklidge System
}

\author{
Chengwei Dong $\mathbb{D}$, Lian Jia, Qi Jie $\mathbb{i}$, and Hantao Li $\mathbb{D}$ \\ Department of Physics, North University of China, Taiyuan 030051, China \\ Correspondence should be addressed to Chengwei Dong; dongchengwei@tsinghua.org.cn, Qi Jie; jieqi@nuc.edu.cn, and Hantao Li; \\ lihantao@nuc.edu.cn
}

Received 20 May 2021; Revised 11 July 2021; Accepted 14 August 2021; Published 27 August 2021

Academic Editor: Eric Campos

Copyright ( $) 2021$ Chengwei Dong et al. This is an open access article distributed under the Creative Commons Attribution License, which permits unrestricted use, distribution, and reproduction in any medium, provided the original work is properly cited.

\begin{abstract}
To describe and analyze the unstable periodic orbits of the Rucklidge system, a so-called symbolic encoding method is introduced, which has been proven to be an efficient tool to explore the topological properties concealed in these periodic orbits. In this work, the unstable periodic orbits up to a certain topological length in the Rucklidge system are systematically investigated via a proposed variational method. The dynamics in the Rucklidge system are explored by using phase portrait analysis, Lyapunov exponents, and Poincaré first return maps. Symbolic encodings of the periodic orbits with two and four letters based on the trajectory topology in the phase space are implemented under two sets of parameter values. Meanwhile, the bifurcations of the periodic orbits are explored, significantly improving the understanding of the dynamics of the Rucklidge system. The multipleletter symbolic encoding method could also be applicable to other nonlinear dynamical systems.
\end{abstract}

\section{Introduction}

Chaos, as a common nonlinear phenomenon, has become a popular research interest over the last half-century within diverse and multidisciplinary scientific communities. The classic Lorenz system, which describes atmospheric convection, is a well-known chaotic deterministic system and was an important milestone in the history of chaos $[1,2]$. Since then, various chaotic systems with special dynamic behavior have been constructed by many researchers [3-5]. For example, the Rössler system was proposed with only one nonlinear term [6], the Chen system was constructed using a chaos anticontrol method [7], and the Lü system was further found [8], which is the key bridge between the Lorenz system and the Chen system. Some chaotic systems were also presented using exhaustive computer methods by Sprott [9-11]. Very recently, in the presence of an external periodic perturbation, the periodic and chaotic motions of the perturbed modified equal width-Burgers equation [12] and the perturbed dual power Zakharov-Kuznetsov-Burgers equation [13] were explored.
In fluid mechanics, the problem of $2 \mathrm{D}$ convection in a horizontal layer of a Boussinesq fluid with lateral constants was explored by Rucklidge [14]. The Rucklidge system is a classical chaotic system that shows extremely rich dynamic behavior, and it has potential applications in the field of secure communications. Many theoretical and numerical results for the Rucklidge system have been investigated in the literature. In [15], the synchronization of fractionalorder Rucklidge systems was studied based on three different schemes. Hopf bifurcations of the Rucklidge system were investigated by an analytical method in [16], and the existence of connecting orbits was also proven. In [17], the authors designed and simulated a chaotic oscillator and studied the synchronization and masking communication circuits of the Rucklidge attractor. Small-amplitude limit cycles in the Rucklidge system were discussed in [18], and bifurcation analysis at the equilibria was performed by calculating the second Lyapunov coefficient or the first Lyapunov value at the stability boundary [19]. Control of the chaotic behavior of the Rucklidge system was analyzed through various control techniques in [20-22]. In [23], the 
analytic integrability of the Rucklidge system was explored. Recently, a novel method to generate double-scroll chaotic attractors from the Rucklidge system via the arctangent function series was proposed in [24].

Unstable periodic orbits of turbulent flows have recently drawn considerable attention in the research of fluid dynamics because they provide the building blocks of disordered dynamics [25]. It is hoped that unstable periodic orbits can be used as fundamental components of turbulent motion. In a chaotic system, unstable periodic orbits also play important roles in the analysis of dynamic behavior. In addition to being densely embedded around strange attractors [26], the dynamical average values of chaotic systems can be calculated by cycle expansions $[27,28]$, which express long-time averages over chaotic orbits in terms of averages over periodic orbits in a hierarchical manner. If the system is hyperbolic, long periodic orbits are shadowed by short ones, and cycle expansions converge rapidly with increased cycle length $[29,30]$.

Unstable periodic orbits in nonlinear dynamical systems are never easy to extract, especially in high-dimensional chaotic systems, and various design methods have emerged to locate periodic orbits in the past several decades [31]. Recent work focused on finding periodic orbits quickly and accurately, and a variational method, introduced by Lan and Cvitanović, can be used to calculate periodic orbits conveniently [32]. Another problem is that even if some periodic orbits are calculated, it is still uncertain whether all the short periodic orbits are found. A key step for solving this problem is to build appropriate symbolic dynamics, which can encode all the possible orbits and their topological layout [33-35]. Symbolic dynamics are thoroughly investigated and usually used for 1D unimodal maps. However, it is considerably harder to construct the symbolic dynamics in maps with multiple critical points or $2 \mathrm{D}$ maps. In the aforementioned research work, only a few scattered periodic orbits in the Rucklidge system were extracted using the existing numerical methods. Due to the lack of effective establishment of appropriate symbolic dynamics, there is no clue as to how many periodic orbits exist and how they are related to each other. In order to describe and analyze the unstable periodic orbits of the Rucklidge system, in this paper, a general scheme is put forward to build diverse symbolic encodings of periodic orbits based on orbit topology in the phase space to systematically classify all found periodic orbits.

The main contributions and novelty of this paper are summarized as follows. (1) We systematically investigate the short unstable periodic orbits up to a certain topological length in the Rucklidge system through a proposed variational method. (2) We develop a topological classification methodology and construct diverse symbolic encodings of unstable periodic orbits with two and four letters in the Rucklidge system for two sets of parameters. Compared with the two-letter symbolic encoding approach, the multipleletter symbolic encoding method can serve as a proof of concept that can fuel studies of more involved complex systems. (3) We explore the pitchfork bifurcation, perioddoubling bifurcation, and saddle-node bifurcation of periodic orbits in the Rucklidge system by the homotopy evolution approach.

The rest paper is organized as follows. In Section 2, the dynamics of the Rucklidge system are discussed, and the use of classical nonlinear analysis tools helps to confirm its chaotic behavior. In Section 3, to locate the unstable periodic orbits in the Rucklidge chaotic system effectively, a variational method is introduced. Section 4 presents the main results of this work, and a general method for the classification of periodic orbits in a chaotic system is developed. Diverse symbolic encodings of periodic orbits in the Rucklidge system are constructed for two different sets of parameters $(a, b)=(2,6.7)$ and $(a, b)=(2,9.3)$. In Section 5 , various bifurcations in the Rucklidge system are discussed, which could significantly improve the understanding of the dynamics of the Rucklidge system. Some conclusions are drawn in the last section.

\section{Dynamics of the Rucklidge System}

The Rucklidge system is a model of a double convection process, which models convection in an imposed vertical magnetic field and in a uniformly rotating layer of fluid, with the motion confined to tall thin rolls. The Rucklidge system has a similar form to the Lorenz system, but it does not belong to the category of the generalized Lorenz system. The system with two quadratic nonlinearities is described by the following ordinary differential equations [14]:

$$
\begin{aligned}
& \frac{\mathrm{d} x}{\mathrm{~d} t}=-a x+b y-y z, \\
& \frac{\mathrm{d} y}{\mathrm{~d} t}=x, \\
& \frac{\mathrm{d} z}{\mathrm{~d} t}=y^{2}-z,
\end{aligned}
$$

where $x, y, z$ are state variables, and the two unfolding parameters $a$ and $b$ are positive real constants. In these state variables, $y$ is proportional to the convective amplitude, $x$ is the rate of change of $y$, and $z$ is proportional to the horizontal average temperature. The volume contraction of equation (1) can be evaluated by $\nabla \cdot V=\partial \dot{x} / \partial x+\partial \dot{y} / \partial y+\partial \dot{z} / \partial z=-a-1$, which means that the system is dissipative when $a>-1$. Thus, trajectories of the Rucklidge system eventually converge to a set of measure zero at an exponential rate of $e^{-a-1}$, and the asymptotic motion at the time through the flow will settle onto an attractor.

As shown in equation (1), the Rucklidge system is symmetric with respect to the $z$-axis. Letting the left-hand side of system (1) equal zero, for the positive parameters $b$, three equilibrium points of system (1) can be obtained:

$$
\begin{aligned}
& E_{0}:(0,0,0), \\
& E_{1}:(0, \sqrt{b}, b), \\
& E_{2}:(0,-\sqrt{b}, b) .
\end{aligned}
$$

The Jacobian matrix of equation (1) is 


$$
A=\left(\begin{array}{ccc}
-a & b-z & -y \\
1 & 0 & 0 \\
0 & 2 y & -1
\end{array}\right) .
$$

For the typical parameters $(a, b)=(2,6.7)$, the Rucklidge system is in a state of chaos. Figure 1 shows the chaotic trajectories of the system in different phase spaces obtained by numerical simulations with the initial condition $x_{0}=2$, $y_{0}=2$, and $z_{0}=2$. The eigenvalues for the parameters mentioned above can be calculated from the Jacobian matrix (3), as follows:

$$
\begin{aligned}
E_{0}:\left(\lambda_{1}, \lambda_{2}, \lambda_{3}\right) & =(-3.7749,1.7749,-1.0000), \\
E_{1,2}:\left(\mu_{1} \pm i \omega_{1}, \lambda_{3}\right) & =(0.2577 \pm 1.9353 i,-3.5154) .
\end{aligned}
$$

The eigenvalues indicate that $E_{0}$ is a saddle and $E_{1,2}$ are two unstable saddle-focus points. Furthermore, the chaotic trajectories have two wings alternately rotating near the two nonhyperbolic fixed points, and the rotation period calculated from the eigenvalues above is $T=\left(2 \pi / \omega_{1}\right)=3.2449$. Correspondingly, the Floquet multipliers for the fixed point $E_{1,2}$ are $\Lambda^{(1)}=2.3076$ and $\Lambda^{(3)}=1.1116 \times 10^{-5}$.

In a chaotic system, the change of the parameters affects the stability of the system, causing the system to be in a different state. To obtain the dynamical modes of system (1) for different parameters, the division diagram can be drawn. Figure 2 presents a global view of the $(a, b)$ parameter space, indicating different dynamic behaviors characterized by different tonalities. The maximum Lyapunov exponents are calculated to obtain the pseudocolored map on a $120 \times 120$ grid of parameters $(a, b)$, where the colors are related to the magnitude of the maximum Lyapunov exponent. The deep blue color corresponds to the most negative largest Lyapunov exponent, which represents a fixed point, yellow corresponds to a zero value of the largest Lyapunov exponent, which represents a periodic orbit, and red corresponds to the most positive largest Lyapunov exponent, which represents chaos.

For different parameter values, $(a, b)=(2,9.3)$, which correspond to a chaotic state (see Figure 2), the three eigenvalues of the fixed points $E_{1,2}$ are $\lambda_{1,2}=0.2243 \pm 2.4692 i$ and $\lambda_{3}=-3.4486$. Thus, $E_{1,2}$ also correspond to two saddlefoci for these parameters. The fractional dimension of the system, which indicates the complexity of the attractor, can also be calculated. The Kaplan-Yorke dimension of system (1) is defined by

$$
D_{K Y}=j+\frac{1}{\left|\lambda_{j+1}\right|} \sum_{i=1}^{j} \lambda_{i},
$$

where $j$ denotes the largest integer satisfying $\sum_{i=1}^{j} \lambda_{i} \geq 0$ and $\sum_{i=1}^{j+1} \lambda_{i}<0$. Thus, the Kaplan-Yorke dimension for the parameters $(a, b)=(2,9.3)$ is $D_{K Y}=2+((0.2819-0.0010) / \mid$ $-3.2809 \mid)=2.0856$. Since the Kaplan-Yorke dimension is a noninteger between 2 and 3, it indicates a strange attractor. The chaotic time series and the power spectrum with continuous broadband characteristics also clearly support this conclusion, as shown in Figure 3.
The strange attractor of the Rucklidge system is made up of numerous unstable periodic orbits, and the first return map can help to make a preliminary prediction for the selection of symbolic encoding of periodic orbits. We choose the above two groups of parameters, since their first return map diagrams were significantly different, which indicates that the periodic orbits of the system will have different complexities. Figure 4(a) displays the first return map of the Rucklidge system for the parameters $(a, b)=$ $(2,6.7)$ for the special Poincaré section $z=6.7$. The map is a sheet of the point set with a certain hierarchical structure. It is unimodal for this parameter, which implies that all the unstable periodic orbits can be encoded with two letters. However, it is worth noting that the first return map is not unimodal for the parameters $(a, b)=(2,9.3)$, as shown in Figure 4(b). It presents a trimodal return map, and four branches are present, which implies that multiple symbol sequences are needed to encode the cycles. In this case, the topological structures of the unstable periodic orbits in the phase space become more complicated, which is worthy of further study. To calculate the unstable cycles effectively, the variational method is used for the calculations [32], and the applicability of this method is verified by various chaotic systems [36-38], including dissipative or Hamilton systems. We will review the variational calculation method in the next section.

\section{Variational Calculation Method}

Periodic orbits are a foundation of classical and quantum chaotic dynamics. When we explore and seek to understand the dynamics of a chaotic system, good knowledge of the periodic orbits is essential, because they form the skeleton of the overall dynamics, especially for any chaotic invariant set [39]. Many numerical methods for calculating periodic orbits have been devised [31], such as the inverse iteration and Newton methods, which are suitable for finding cycles for 1D maps, the multipoint shooting method, which is applicable for determining long cycles for a continuous time flow, and the variational method we used in this study. The variational method retains the robustness of the multipoint shooting method, which can converge to the real periodic orbit rapidly when the initial loop guess is appropriate. In the application of the variational method, a virtual evolution equation was derived in [32]:

$$
\frac{\partial^{2} \widetilde{x}}{\partial s \partial \tau}-\lambda A \frac{\partial \widetilde{x}}{\partial \tau}-v \frac{\partial \lambda}{\partial \tau}=\lambda v-\widetilde{v},
$$

where $\widetilde{v}=(\mathrm{d} \tilde{x} / \mathrm{d} s)$ is the loop velocity vector, which is parameterized by $s \in[0,2 \pi], v$ is the flow velocity vector, $\tau$ is the virtual time, which is related to the number of iterations, $\lambda$ is used to adjust the period, and $A_{i j}=\left(\partial v_{i} / \partial x_{j}\right)$ is the gradient matrix of the velocity field.

A monotonic minimizing cost functional exists when the loop evolves toward the cycle:

$$
F^{2}[(\widetilde{x})] \equiv \frac{1}{2 \pi} \oint_{L(\tau)} \mathrm{d} \widetilde{x}[\widetilde{v}(\widetilde{x})-\lambda v(\widetilde{x})]^{2} .
$$




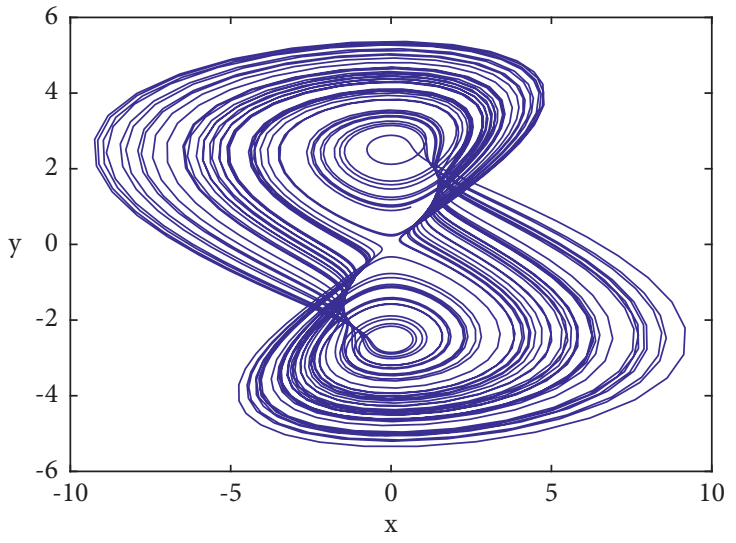

(a)

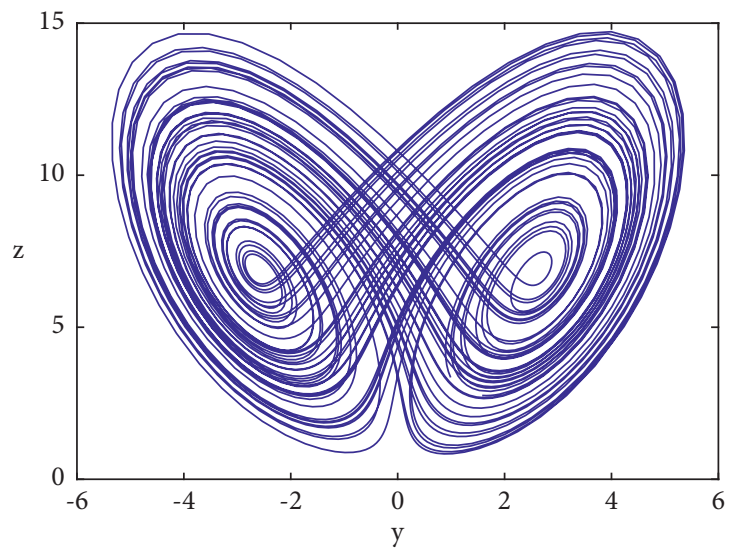

(c)

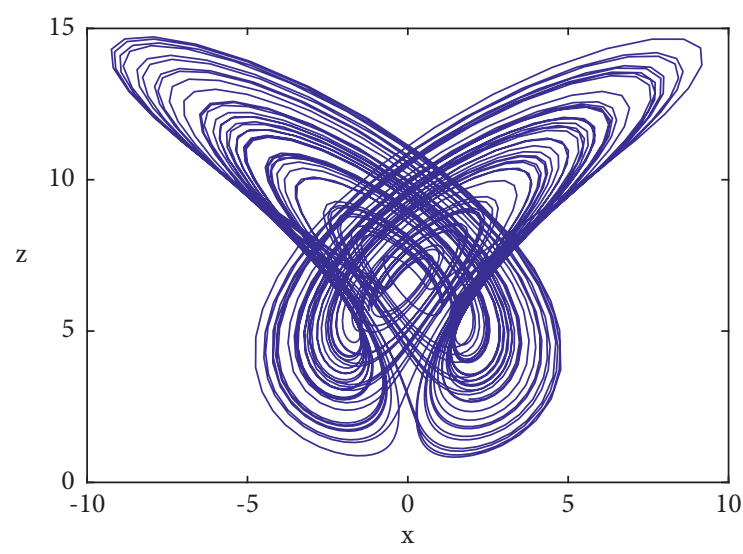

(b)

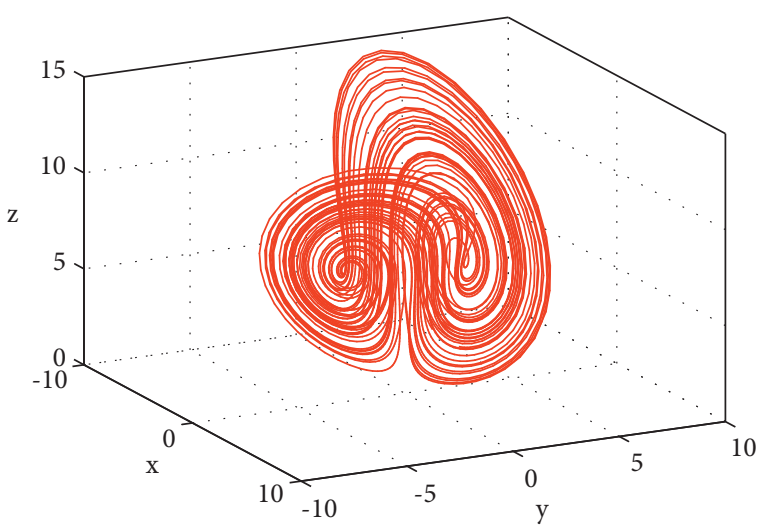

(d)

Figure 1: Phase portrait of the Rucklidge system in different two-dimensional (2D) and three-dimensional $(3 \mathrm{D})$ phase spaces for $(a, b)=$ $(2,6.7)$ from $t=20000$ to 20300 .

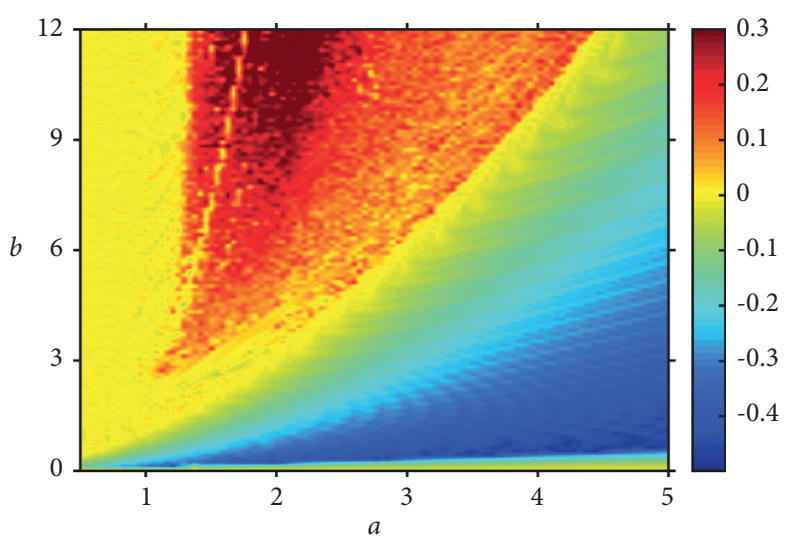

FIGURE 2: Division of parameters $a$ and $b$ by a pseudocolored map.

As the virtual time $\tau$ tends to infinity, the loop guess gradually evolves into the real periodic orbit. Figure 5 displays the schematic diagram of the variational method. Not only the location but also the period of the cycle in the chaotic attractor can be computed by the variational method:

$$
T_{p}=\int_{0}^{2 \pi} \lambda(\tilde{x}(s, \infty)) \mathrm{d} s
$$

For the discretization of a loop, the finite difference scheme is employed: 


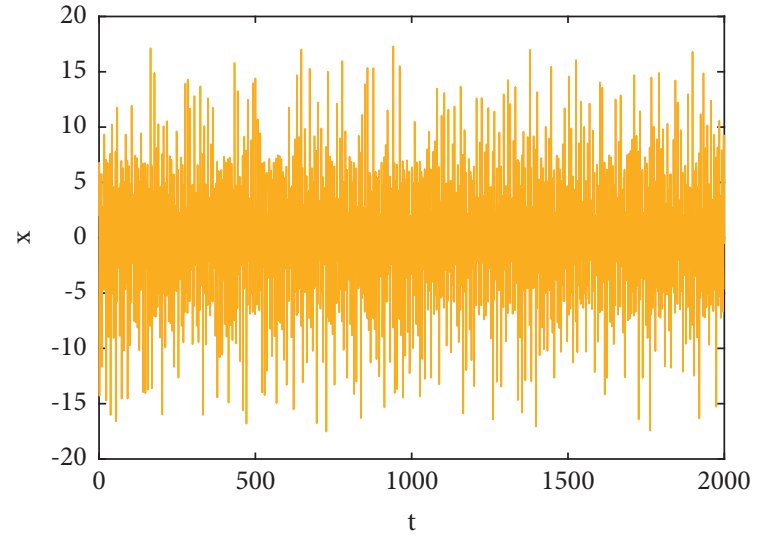

(a)

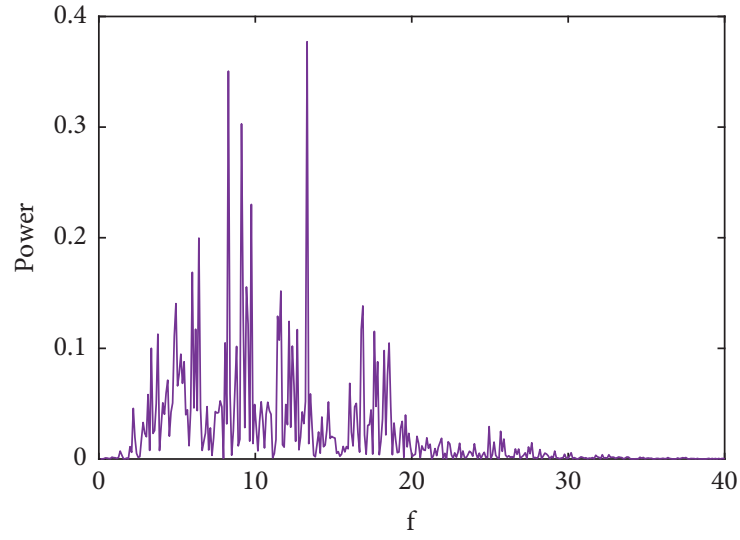

(b)

Figure 3: (a) Chaotic time series of the Rucklidge system for the $x$ signal. (b) Apparently continuous broadband power spectrum. Note: $(a, b)=(2,9.3)$.

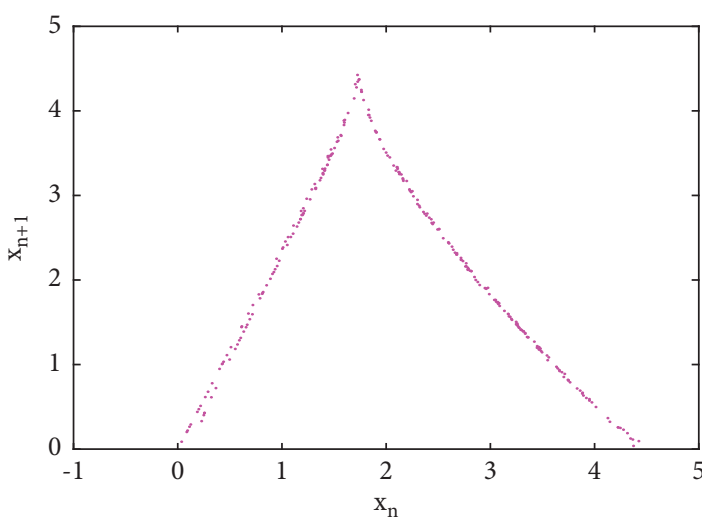

(a)

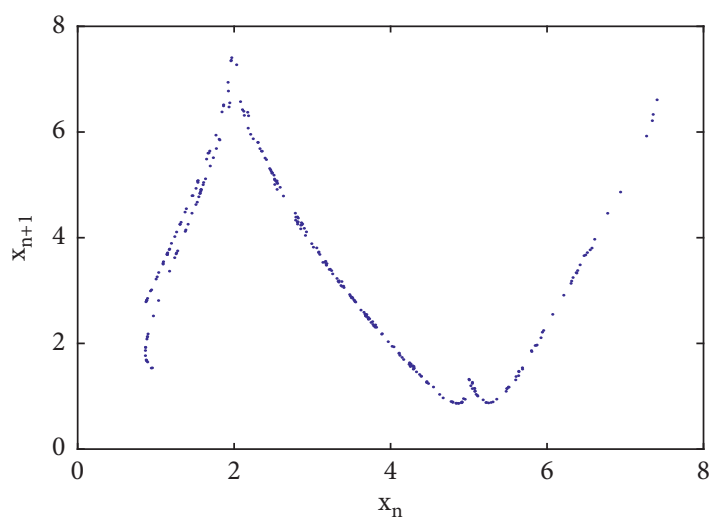

(b)

Figure 4: First return map for Poincaré section: (a) $z=6.7,(a, b)=(2,6,7)$ and (b) $z=9.3,(a, b)=(2,9.3)$.

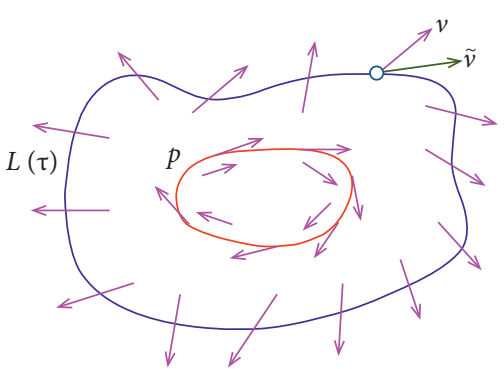

FIgURE 5: Velocity field of a guess loop $\widetilde{v}(\widetilde{x})$ coinciding with the velocity field of the flow $v(\tilde{x})$ when a periodic orbit was found.

$$
\left.\widetilde{v}_{n} \equiv \frac{\partial \widetilde{x}}{\partial s}\right|_{\widetilde{x}=\tilde{x}\left(s_{n}\right)} \approx(\widehat{D} \tilde{x})_{n}
$$

We adopt the five-point approximation:

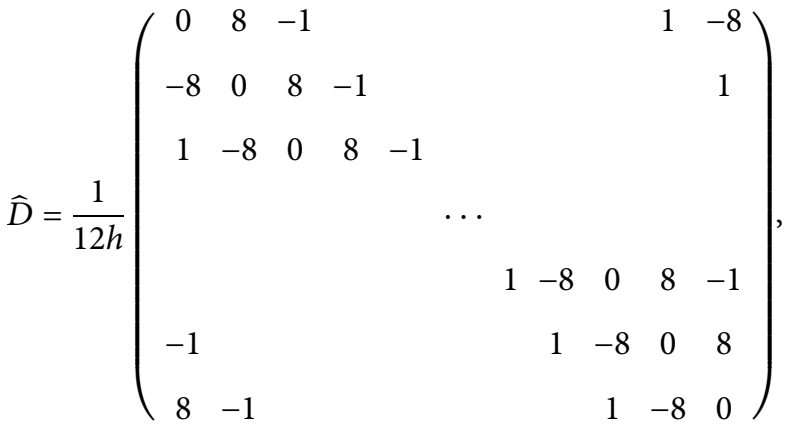

where $h=2 \pi / N$. After discretization, equation (6) becomes

$$
\left(\begin{array}{cc}
\widehat{A} & -\widetilde{v} \\
\widehat{a} & 0
\end{array}\right)\left(\begin{array}{l}
\delta \tilde{x} \\
\delta \lambda
\end{array}\right)=\delta \tau\left(\begin{array}{c}
\lambda \widehat{v}-\widehat{\widetilde{v}} \\
0
\end{array}\right),
$$


where $\widehat{A}=\widehat{D}-\lambda \operatorname{diag}\left[A_{1}, A_{2}, \ldots, A_{N}\right], \widehat{v}=\left(v_{1}, v_{2}, \ldots, v_{N}\right)^{t}$, $\widehat{\widetilde{v}}=\left(\widetilde{v}_{1}, \widetilde{v}_{2}, \ldots, \widetilde{v}_{N}\right)^{t}$, and $\widehat{a}$ is an $N d$-dimensional row vector, which restricts the coordinate variations. In actual calculations, a banded lower-upper decomposition scheme can be used to accelerate the computation, and the Woodbury formula is adopted to treat the cyclic and border terms in the calculations [40]. Furthermore, as the virtual time steps are not very important in some cases, larger time steps may be chosen to carry out the Euler integration, thus enabling an efficient search for the real periodic orbits.

With proper modification, the variational method can also be used to calculate the invariant tori and connecting orbits in a nonlinear dynamical system [41, 42]. The method can be used not only to calculate the cycles with certain parameter values but also to investigate the deformation of the periodic orbits when the parameters change [43]. In Section 5, we will use this approach to analyze the bifurcation behavior in the Rucklidge system.

\section{Topological Classification of Periodic Orbits in the Rucklidge System}

In this section, we utilize the variational method to calculate the periodic orbits and build appropriate symbolic encodings for the cycles found in the Rucklidge system. After several short periodic orbits are located, we seek insights into the encircling behavior of the orbit and propose a systematic methodology for the taxonomy. We present the main results of this paper: diverse symbolic encodings with two and four letters are successfully established in the Rucklidge system for two groups of parameters, which demonstrates that the topological classification method is effective in the encoding of periodic orbits. The search and classification procedure employed herein can also be applied to other low-dimensional dissipative chaotic systems.

\subsection{Symbolic Encoding of Periodic Orbits with Two Letters for} $(a, b)=(2,6.7)$. We calculated the unstable periodic orbits for parameter values of $(a, b)=(2,6.7)$ for the Rucklidge system. In the application of the variational method, there are many ways to initialize the problem [44]. First, a conjecture loop that is suitable for periodic orbit calculations is a prerequisite. To gain insight into the general shapes of the periodic orbits, the classical fourth-order Runge-Kutta algorithm was first used to integrate the dynamical system numerically, and the fast Fourier transform of the nearly recurring orbital fragment was computed. The high-frequency modes were then removed, and the inverse fast Fourier transform to the state space yielded a smooth loop guess that could be intercepted to initialize in the numerical calculations. It is convenient and effective in locating periodic orbits with this initialization approach.

The Poincare first return map with two monotonic branches for this set of parameters implies that only two symbols are required to realize the partitioning of the intervals. An interesting approach is proposed to establish symbolic encoding by taking advantage of the topologies of the orbits. Figure 6(a) shows the shortest cycle, which had a simple topological structure that was found via the variational method, where the projection is onto the $y-z$ plane. Figure 6(b) shows the found cycle and the two equilibria $E_{1}$ and $E_{2}$. The symbolic encoding of periodic orbits was established as follows:

(1) The orbital segment swirling around the left equilibrium $E_{2}$ once is denoted by the symbol 0

(2) The orbital segment swirling around the right equilibrium $E_{1}$ once is denoted by the symbol 1

(3) Longer periodic orbits are encoded by using the 0 and 1 orbital fragments as basic building blocks

Thus, the periodic orbit in Figure 6(b) is cycle 01. However, we found that cycles 0 and 1 did not exist, so they were pruned. Figure 7 (a) displays cycle 001, which represents orbit fragments that circulate twice around the left fixed point and once around the right. Figure 7(b) displays cycle 0011 , which is composed of two 0 orbital fragments and two 1 orbital fragments. Figures 7 (c) and 7(d) display two cycles with a topological length of 5 . Using the defined symbolic rule, cycles up to a topological length of 5 are sought, and they are ordered according to their topological length in Table 1. The two simplest orbital segments are regarded as basic building blocks to construct longer periodic orbits based on the symbolic encoding, and the periodic orbits with any topological length can be calculated. Furthermore, the symmetry property of equation (1) represents the commutative symmetry 0 and 1 of the symbol sequence. Consequently, the symmetric counterpart of cycle 001 is 011 , and they have the same period. Cycle 01 is conjugated with itself, as is cycle 0011. As a result, no other cycle has the same period as cycles 01 and 0011.

4.2. Symbolic Encoding of Periodic Orbits with Four Letters for $(a, b)=(2,9.3)$. We now investigate the unstable periodic orbits in the Rucklidge system for the parameters $(a, b)=(2,9.3)$. To the best of our knowledge, there is little information about the unstable cycles for these parameter values, and investigations of the more complex topological structure have rarely been reported. For this reason, our work will expand the topological classification approach to build up the appropriate symbolic encodings. After a few attempts, we acquired some short periodic orbits, and the loop guess was initialized as described below. We numerically integrated equation (1), then extracted the nearly closed orbits for different shapes, and manually connected them to form closed orbits to initialize the search. This initialization method provides a good loop for the calculation of short unstable periodic orbits in the Rucklidge system. Figure 8(a) displays one of the shortest periodic orbits with a relatively simple topological structure, which revolves one turn around the left fixed point. There are four different situations in which the orbit circulates once around the left and right equilibria, and three of these periodic orbits are shown in Figures 8(b)-8(d).

The symbolic encoding using four letters to label the cycles under this set of parameters is established as follows: 


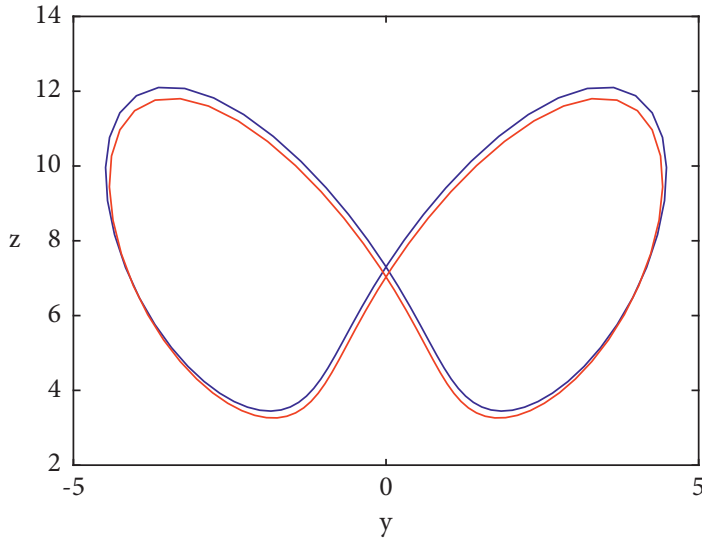

(a)

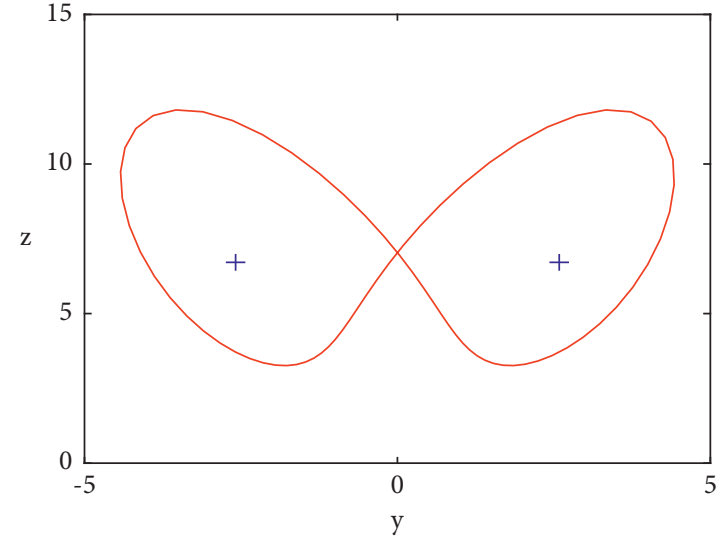

(b)

FIGURE 6: (a) Shortest unstable periodic orbit in the Rucklidge system for $(a, b)=(2,6.7)$. The blue line represents a loop conjecture, and the red line represents a real cycle. (b) Projection of cycle 01 and the locations of two equilibria, which are marked as “+”.

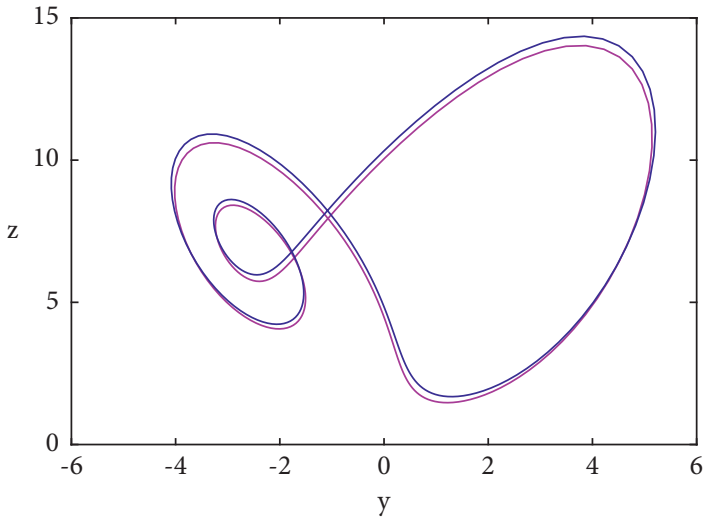

(a)

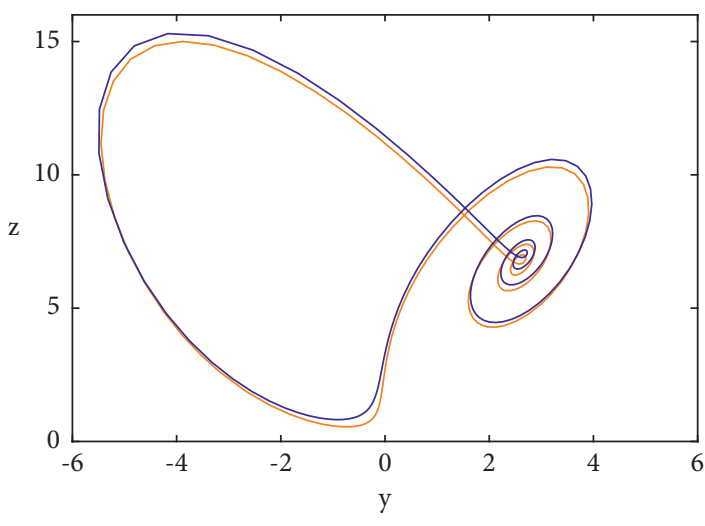

(c)

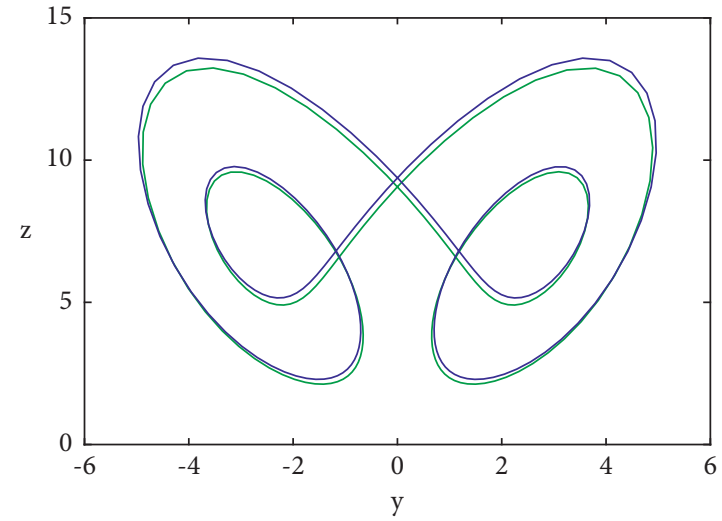

(b)

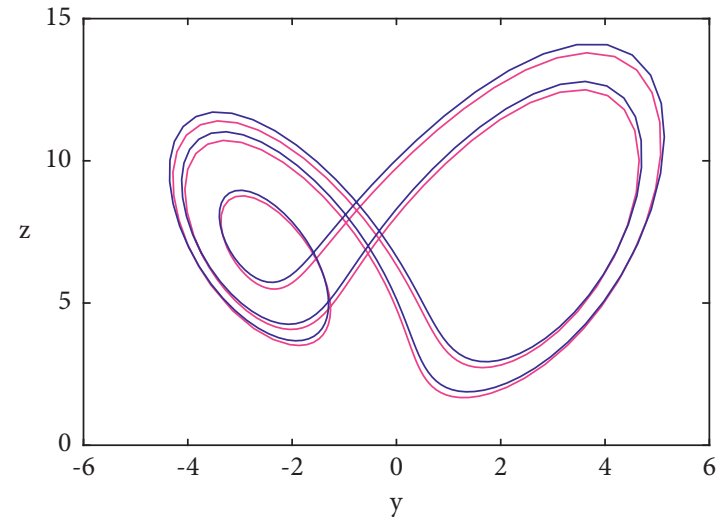

(d)

Figure 7: Four unstable cycles in the Rucklidge system for $(a, b)=(2,6.7)$ : (a) cycle 001, (b) cycle 0011, (c) cycle 01111 , and (d) cycle 00101. Blue curves represent the initial loop guess used for the calculations. 
TABLE 1: Twelve unstable cycles in the Rucklidge system for $(a, b)=(2,6.7)$. The topological lengths, itineraries, periods, and coordinates of a point on the cycle are listed.

\begin{tabular}{|c|c|c|c|c|c|}
\hline Lengths & Itineraries & Periods & $x$ & $y$ & $z$ \\
\hline \multirow{2}{*}{1} & 0 & - & - & - & - \\
\hline & 1 & - & - & - & - \\
\hline 2 & 01 & 6.694493 & 1.438420 & 0.700711 & 4.976993 \\
\hline \multirow{2}{*}{3} & 001 & 10.502415 & -1.652903 & -3.806968 & 7.132296 \\
\hline & 011 & 10.502415 & 1.652903 & 3.806968 & 7.132296 \\
\hline \multirow{3}{*}{4} & 0001 & 14.350272 & -1.767303 & -3.642999 & 6.739171 \\
\hline & 0011 & 13.797828 & 0.786049 & -0.783689 & 5.196282 \\
\hline & 0111 & 14.350272 & 1.767303 & 3.642999 & 6.739171 \\
\hline \multirow{6}{*}{5} & 00001 & 18.051169 & -0.626952 & -2.328136 & 5.666088 \\
\hline & 00011 & 17.549754 & 0.079300 & -3.044837 & 7.467589 \\
\hline & 00101 & 17.138379 & -2.082257 & -3.670226 & 6.512911 \\
\hline & 00111 & 17.549754 & -0.079300 & 3.044837 & 7.467589 \\
\hline & 01011 & 17.138379 & 2.082257 & 3.670226 & 6.512911 \\
\hline & 01111 & 18.051169 & 0.626952 & 2.328136 & 5.666088 \\
\hline
\end{tabular}

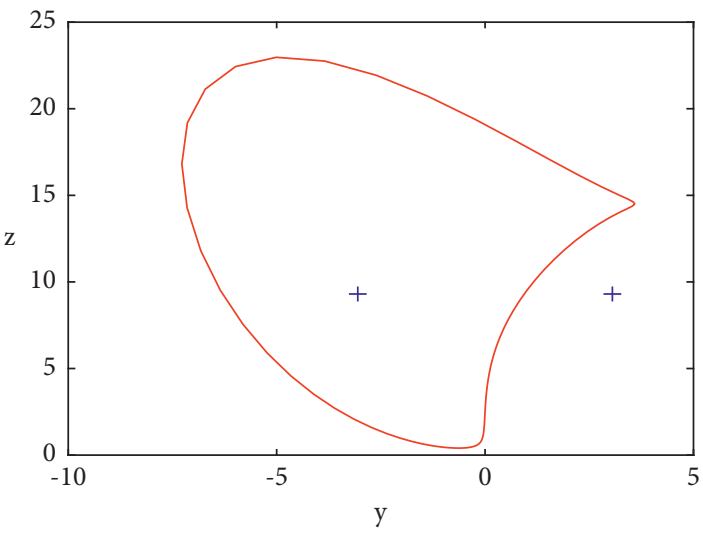

(a)

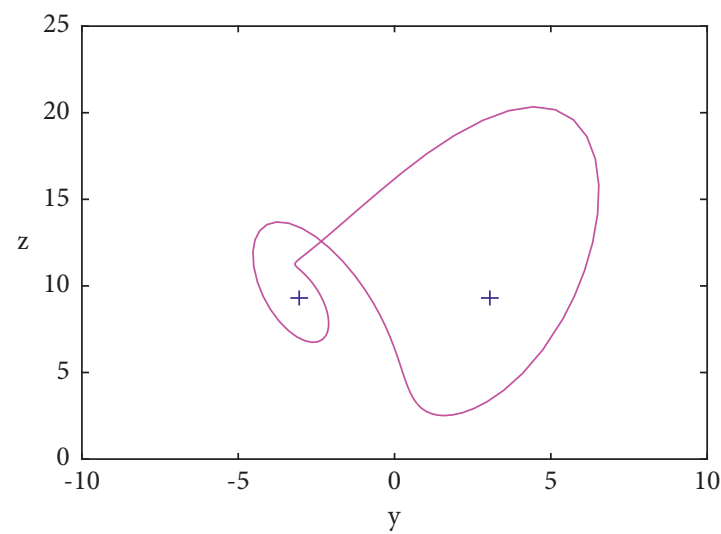

(c)

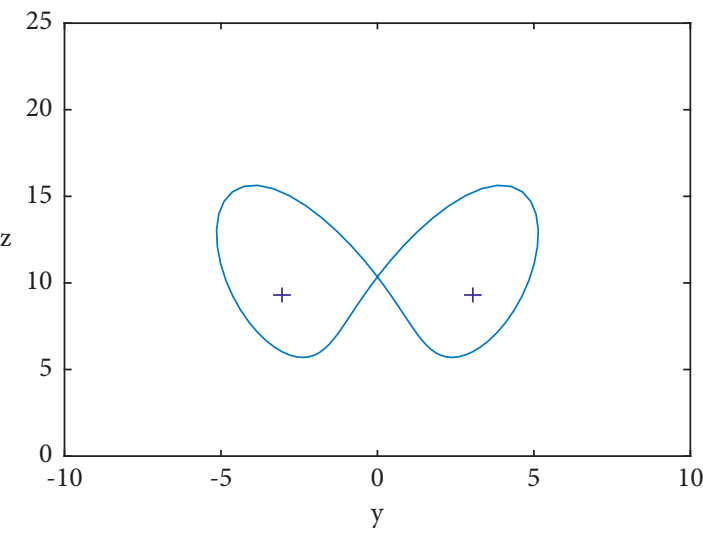

(b)

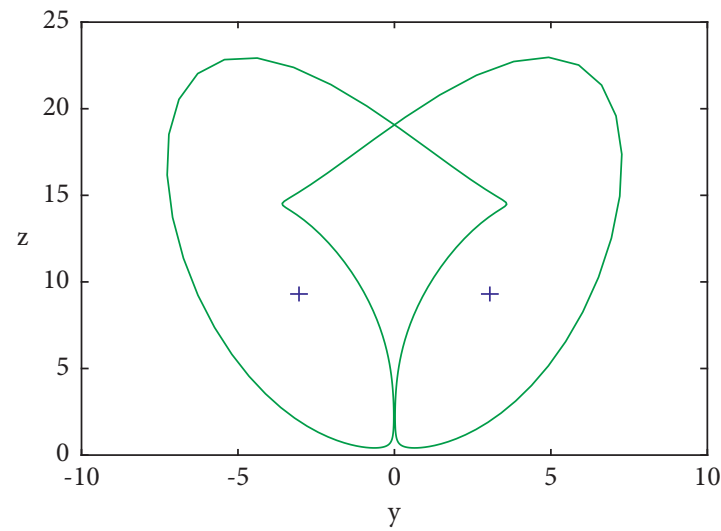

(d)

Figure 8: Continued. 


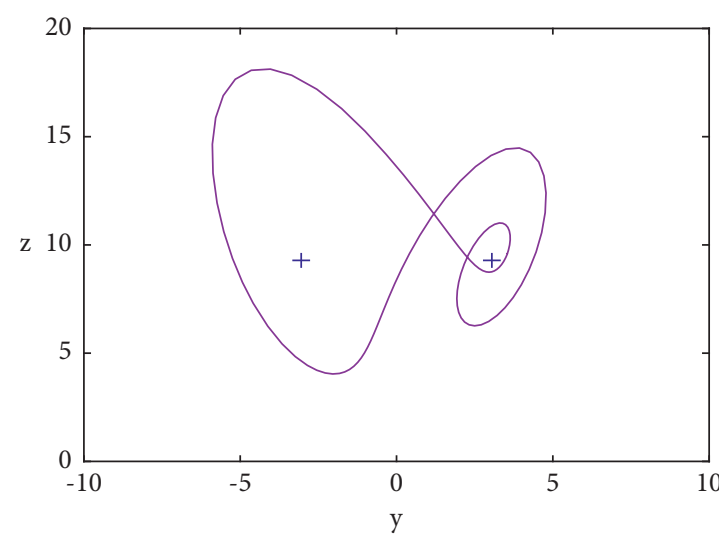

(e)

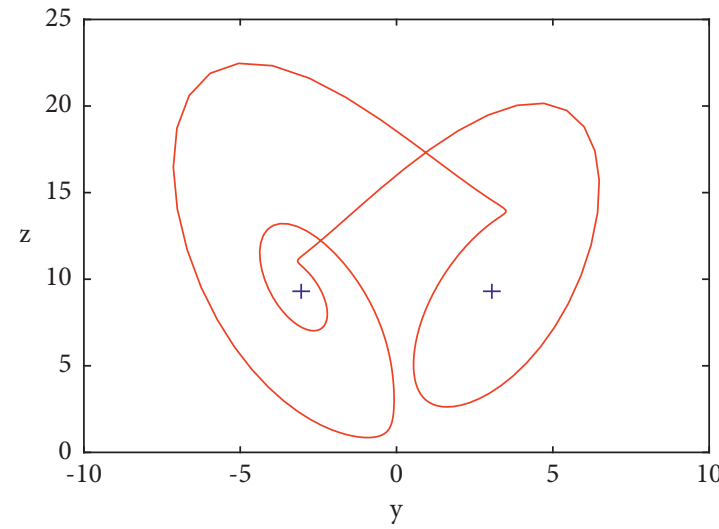

(g)

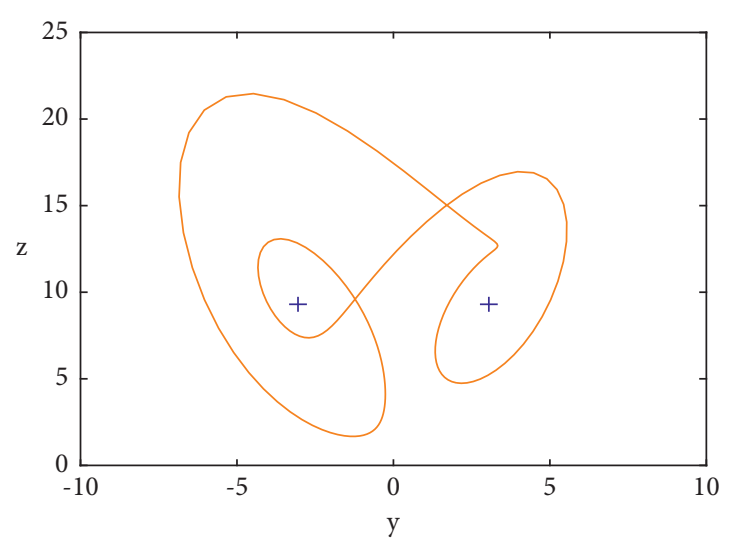

(f)

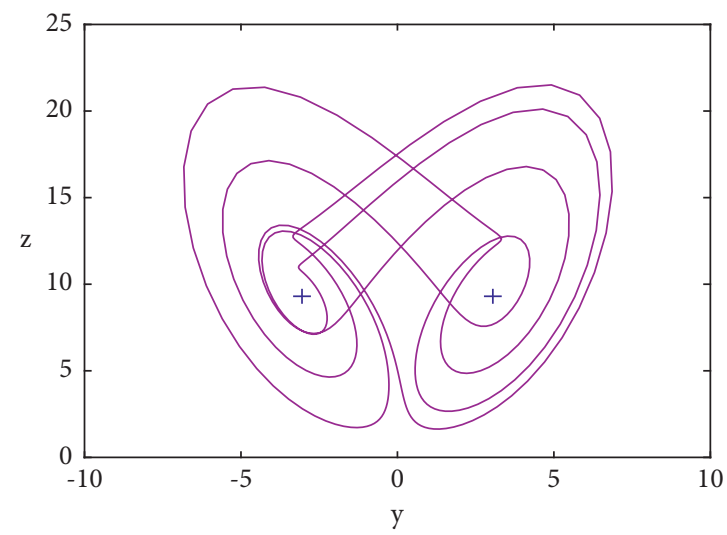

(h)

FiguRe 8: Unstable periodic orbits in the Rucklidge system for $(a, b)=(2,9.3)$ : (a) cycle 2, (b) cycle 01, (c) cycle 03, (d) cycle 23, (e) cycle 011, (f) cycle 021, (g) cycle 023, and (h) cycle 03013021.

(1) Smooth with elliptical shapes, orbital segment swirling around the left or right equilibrium one turn is denoted by the symbol 0 or 1

(2) Orbital segment swirling around the left or right equilibrium one turn in the shape of a raised wing, which has a relatively large extension along the $z$-axis is denoted by the symbol 2 or 3

(3) Complex periodic orbits are encoded by using the above four orbital fragments as basic building blocks

The novel symbolic encoding method means that cycles with topological lengths of 1 are encoded by the symbols 0,1 , 2 , and 3 . We found cycles 0 and 1 were actually pruned, as in the case of the previous parameters. The cycle in Figure 8(a) is marked as cycle 2 , and its symmetric cycle is denoted as cycle 3 . The cycle in Figure 8(b) consists of two relatively small orbital fragments extending in the $z$-axis, so it was labeled as cycle 01 . Utilizing the above four basic building blocks, other cycles can be calculated and classified by the novel symbolic encoding. The cycle in Figure 8(c) consists of one 0 orbital fragment and one 3 orbital fragment, so it is referred to as cycle 03 . Figures $8(\mathrm{e})-8(\mathrm{~g})$ display three cycles with topological lengths of 3 . The topological classification approach employed here shows its effectiveness in encoding cycles. Figure $8(\mathrm{~h})$ shows a long cycle with a topological length of 8. Such complex unstable periodic orbits are difficult to locate without guidance and knowledge of the symbolic encoding. We found a total of 14 unstable periodic orbits with the maximum topological length of 3 and sorted them in Table 2.

Once the unstable periodic orbits are extracted from the chaotic attractor, we can proceed to compute the topological invariants, such as the self-linking number of each cycle and the linking number of the pairs of cycles [45]. The linking number of two cycles indicates the number of turns that are intertwined with each other. Thus, $\operatorname{lk}(A, B)=\mathrm{lk}(B, A)$, and the linking number can be calculated in a simple way:

$$
\operatorname{lk}(A, B)=\frac{1}{2} \sum_{i} \sigma_{i}
$$

where $\sigma_{i}$ denotes the $i$ th signed crossing between the two periodic orbits $A$ and $B$. Therefore, the linking number between cycles $A$ and $B$ can be calculated by counting the number of signed crossings. $\sigma_{i}$ can be taken -1 or +1 depending on whether it is under cross or over cross [45]. There are many applications of the linking number in different disciplines [46, 47]. Correspondingly, the self-linking number for cycle $A$, which represents the linking of a periodic orbit with itself, can be calculated as 
TABLE 2: Unstable cycles in the Rucklidge system for $(a, b)=(2,9.3)$. Listed are the topological lengths, itineraries $p$, periods $T_{p}$, self-linking numbers slk, and linking numbers of other cycles and cycle $2 \mathrm{lk}$.

\begin{tabular}{cccccccccccccc}
\hline Lengths & $p$ & $T_{p}$ & slk & lk & Length & $p$ & $T_{p}$ & slk & lk & $p$ & $T_{p}$ & slk & lk \\
\hline \multirow{4}{*}{1} & 0 & - & - & - & 3 & 011 & 8.388411 & 2 & 0 & 002 & - & - & - \\
& 1 & - & - & - & & 022 & - & - & - & 003 & - & - & - \\
& 2 & 6.697723 & 0 & - & & 033 & - & - & - & 112 & - & - & - \\
\hline & 3 & 6.697723 & 0 & 0 & & 012 & - & - & - & 113 & - & - & - \\
& 01 & 5.518369 & 1 & 0 & & 021 & 10.783775 & 2 & 0 & 132 & 13.547910 & 2 & 0 \\
& 02 & - & - & - & & 031 & - & - & - & 122 & - & - & - \\
2 & 03 & 7.679870 & 1 & 0 & & 023 & 13.547910 & 2 & 0 & 133 & - & - & - \\
& 12 & 7.679870 & 1 & 0 & & 013 & 10.783775 & 2 & 0 & 123 & - & - & - \\
& 13 & - & - & - & & 032 & - & - & - & 223 & 20.044399 & 2 & 0 \\
& 23 & 13.356921 & 1 & 0 & & 001 & 8.388411 & 2 & 0 & 233 & 20.044399 & 2 & 0 \\
\hline
\end{tabular}

$$
\operatorname{slk}(A)=\sum_{i} \sigma_{i}
$$

We also calculated the linking numbers of other cycles and cycle 2 and the self-linking numbers of 14 cycles up to topological length 3 in the Rucklidge system for $(a, b)=(2,9.3)$, as shown in Table 2. The unstable periodic orbits in the Rucklidge system discussed with the current parameter values have more complex topological structures, and thus, a symbolic encoding with four letters must be invoked, which are usually difficult to construct. The topological analysis provides a route to establish effective symbolic encodings and systematically classify all short cycles in a chaotic system. The first return map sometimes may be very complex, and it is difficult to carry out symbolic partitioning. The topological properties of the trajectory can then reveal fascinating dynamic structures, which may not be directly visible from the Poincaré first return map but are important for constructing a symbolic encoding. Determining how to carry out this observation in a high-dimensional state space such as a fluid system remains an open problem. As indicated in this paper, assembling longer cycles with short orbital fragments is a promising candidate to conveniently encode periodic orbits without invoking very complicated topology research.

\section{Bifurcations of Cycles in the Rucklidge System}

The number of cycles and their stability can change when the parameters change, meaning that the bifurcation behavior can be diverse [48]. In this section, we explore three different bifurcations of cycles in the Rucklidge system by introducing a homotopy evolution approach, which shows flexibility in different situations. For the Rucklidge system, when the parameter values change slightly, most short periodic orbits have little deformation unless the system undergoes a bifurcation. Hence, a cycle with specific parameters can be chosen as the initial loop of the next calculation. The advantage of the homotopy evolution approach is that there is no need to select a certain Poincaré section in advance; another advantage is numerical stability due to the use of a continuum of points. Utilizing this initialization approach, only a few iterative calculations are needed to find a new periodic orbit.
5.1. The Pitchfork Bifurcation. Here, we discuss the evolution of unstable cycles when parameter $a$ or $b$ is altered by the variational method, and the homotopy evolution approach can be easily applied to the initial search. We first investigate cycle 01 , which is deformed by varying $a$ while keeping the same value of parameter $b=6.7$. Cycle 01 , which is calculated using the current parameters, is used as the initial guess to calculate the periodic orbit for the next parameters. Figure 9(a) shows the homotopy evolution cases, and Table 3 shows the evolution. Figure 9(b) displays the deformation of cycle 01 with the $b$ value increasing while the value of $a$ was fixed at 2, as summarized in Table 3. Through further analysis of the stability of the cycle, we found that cycle 01 is stable when $a<0.96$. When $a=0.96$, cycle 01 is still stable, but much more weakly. When $a>0.96$, cycle 01 becomes unstable.

We now examine the continuous deformation of cycle 12 for different $a$ values in the same way, and the value of $b$ is fixed at 6.7, as shown in Figure 9(c). Table 4 shows the evolution. We found that cycle 12 appears only when $a>0.96$, and it is stable as the value of $a$ is increased until $a=1.3$, where cycle 12 loses stability. The Lyapunov exponents also show good qualitative agreement with the homotopy evolution results. Figure 10(a) displays the Lyapunov exponent spectrum of the Rucklidge system when the parameters were taken as $(a, b)=(0.96,6.7)$. The Lyapunov exponents converged with time. When $t=1000$, the three Lyapunov exponents of the system are $\lambda_{1}=0.00002$, $\lambda_{2}=-0.05208$, and $\lambda_{3}=-1.90790$. Hence, we concluded that a pitchfork bifurcation occurs at $a=0.96$.

5.2. The Period-Doubling Bifurcation. Pitchfork bifurcation is usually the precursor of period-doubling bifurcation. When $b=6.7$ and $a \in(0.96,1.3)$, we find the symmetric unstable cycle 01 coexists with the asymmetric stable cycles 03 and 12 (see Figure 11). Cycle 12 also becomes unstable when $a$ increases to 1.3 , and there are only unstable cycles in the system. Thus, a period-doubling bifurcation occurs at $a=1.3$, leading to a chaotic state. The bifurcation behavior is also demonstrated by the calculation of the Lyapunov exponents for the parameters $(a, b)=(1.3,6.7)$, which yields $\lambda_{1}=0.0755, \lambda_{2}=-0.0007$, and $\lambda_{3}=-2.3747$, as depicted in Figure 10(b). 


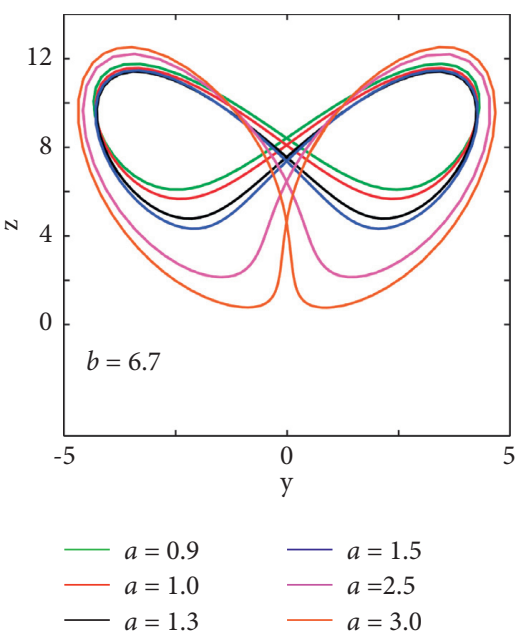

(a)

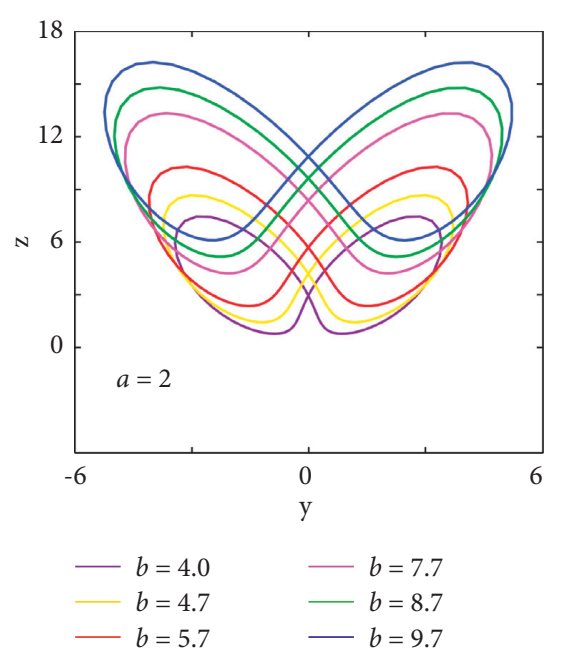

(b)

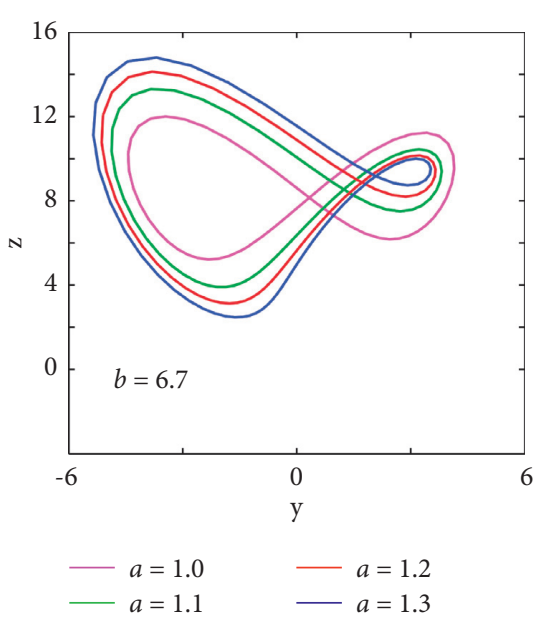

(c)

Figure 9: Homotopy evolution of (a, b) cycle 01 and (c) cycle 12 with different parameter values.

TABle 3: Periods $T_{p}$ of cycle 01 in the Rucklidge system for different parameter values.

\begin{tabular}{lccc}
\hline$a$ & $T_{p}$ & $b$ & $T_{p}$ \\
\hline 0.9 & 4.086584 & 4.0 & 10.374289 \\
1.0 & 4.387094 & 4.7 & 8.702856 \\
1.3 & 5.169756 & 5.7 & 7.461872 \\
1.5 & 5.626114 & 7.7 & 6.147916 \\
2.5 & 7.973592 & 8.7 & 5.728579 \\
3.0 & 10.738449 & 9.7 & 5.391508 \\
\hline
\end{tabular}

TABle 4: Periods $T_{p}$ of cycle 12 and cycle 2 in the Rucklidge system for different $a$ values.

\begin{tabular}{lccc}
\hline$a$ & $T_{p}$ & $a$ & $T_{p}$ \\
\hline 1.0 & 4.372470 & 1.05 & 3.777130 \\
1.1 & 4.498264 & 1.1 & 3.811609 \\
1.2 & 4.656939 & 1.6 & 4.372044 \\
1.3 & 4.866583 & 1.8 & 4.880867 \\
\hline
\end{tabular}

The bifurcation diagrams obtained by varying parameter $a$ or $b$ are calculated to detect the chaotic transition. Figure 12(a) shows the bifurcation diagram versus parameter $b$ for $a=2$ with the initial value $(1,1,1)$ using a local maxima approach, and its corresponding largest Lyapunov exponents calculated numerically by the typical Benettin method are presented in Figure 13(a). Figure 12(b) displays the bifurcation diagram versus parameter $a$ for $b=6.7$ with the same initial value. The bifurcation diagrams coincide well with the different dynamic behaviors for the various parameters shown in Figure 2. The bifurcation diagram in Figure 12(b) shows a period-1 oscillation followed by a process of period-doubling to chaos interleaved with periodic windows, which was also verified by our bifurcation conclusions presented above. Furthermore, the corresponding largest Lyapunov exponents spectrum in Figure 13(b) also reflects this conclusion.
5.3. The Saddle-Node Bifurcation. In the previous discussion, we investigated the bifurcations based on the periodic orbits with a topological length of 2 . Now, we use periodic orbits with a topological length of 1 to explore the saddle-node bifurcation. Here, we fix the parameter $b=9.3$ while letting $a$ vary to study the continuous deformation of cycles 2 and 3 . Figure 14(a) shows the homotopy evolution of cycle 2, and Table 4 lists the numerical results. The four periodic orbits shown in Figure 14(a) are all labeled as cycle 2 because their topological structures are the same; that is, each cycle only rotates around the left fixed point once. Although the periodic orbits with blue and red curves both present a figure- 8 shape, which resemble the cycles with a topological length of 2 , due to the fact that the $z$ coordinate of the right fixed point is 9.3, they actually do not rotate around the right fixed point one turn. By symmetry, cycle 3 also undergoes a similar deformation (see Figure 14(b)). Through the above calculations, we find that the shapes of cycles 2 and 3 become increasingly similar to the decrease in the $a$ value, and they no longer exist for some parameter values. For example, when using the variational method to calculate cycles 2 and 3 , there is no convergence when $a<1.05$. The two cycles collide at the critical value and vanish together after, which means that the system undergoes a saddle-node bifurcation of periodic orbits at $a=1.05$. Due to the possible bifurcations of periodic orbits, when a new set of parameters is taken, it is worth noting that some of the cycles found previously may no longer exist, and new cycles corresponding to the new period may arise. Therefore, if the parameters in the Rucklidge system vary, symbolic encoding may also need to change accordingly.

Finally, according to the results presented above, we investigate the evolution behavior of the orbital period as the parameters changed. The quadratic curve fitting method is used to explore the corresponding relationship between the period of cycle 2 and the variable $a$ (see Figure 14(c)), which is expressed by 

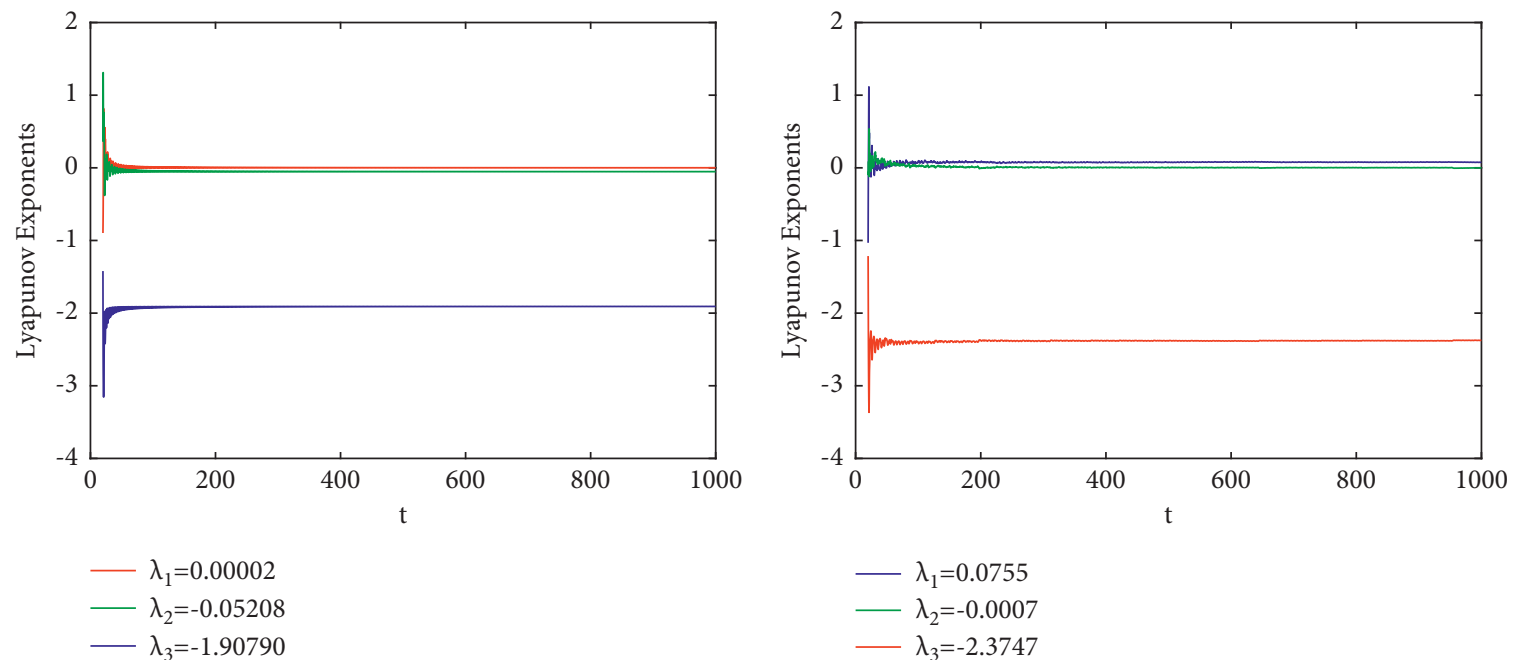

(a)

(b)

FIgURe 10: Lyapunov exponents spectrum of the Rucklidge system. (a) $(a, b)=(0.96,6.7)$ and $(b)(a, b)=(1.3,6.7)$.

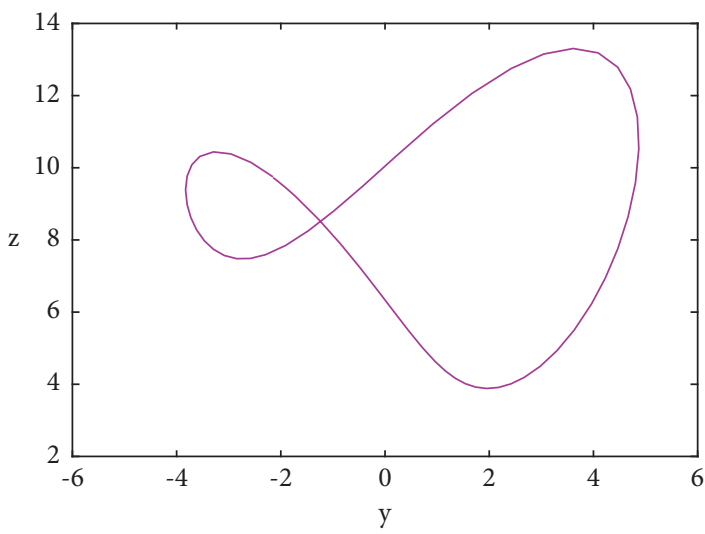

(a)

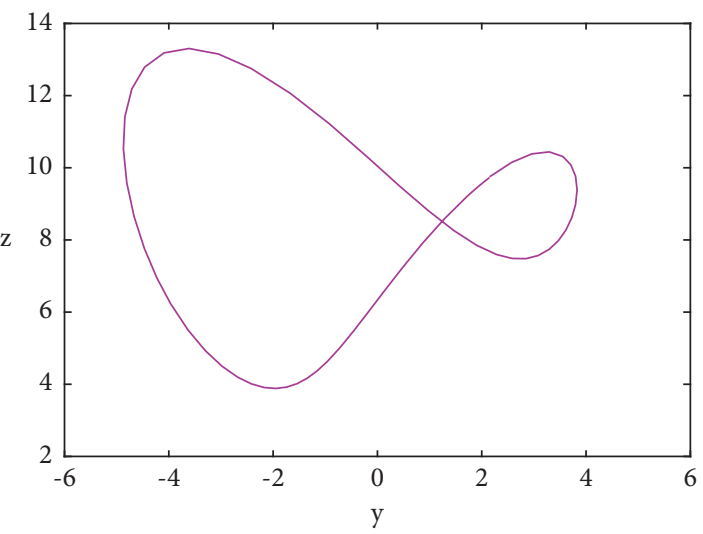

(b)

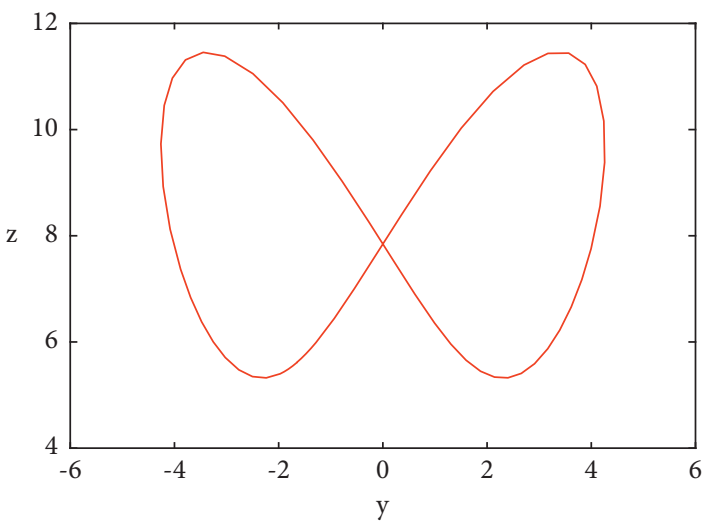

(c)

Figure 11: (a) Stable periodic orbit 03, (b) stable periodic orbit 12, and (c) unstable periodic orbit 01 in the Rucklidge chaotic system for $(a, b)=(1.1,6.7)$. 


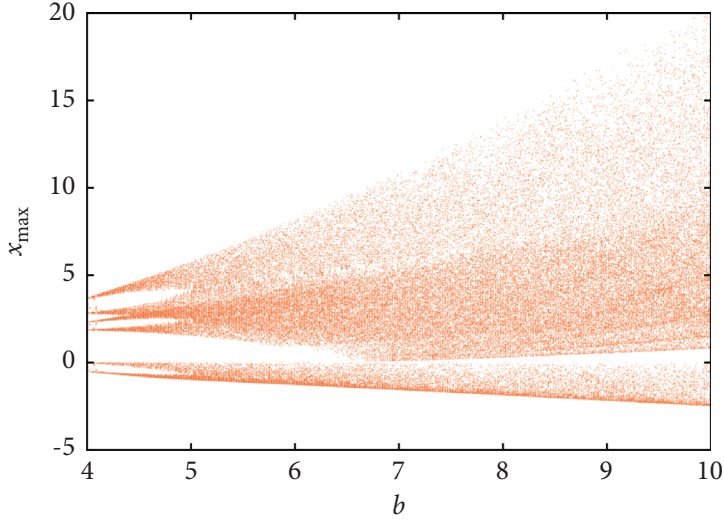

(a)

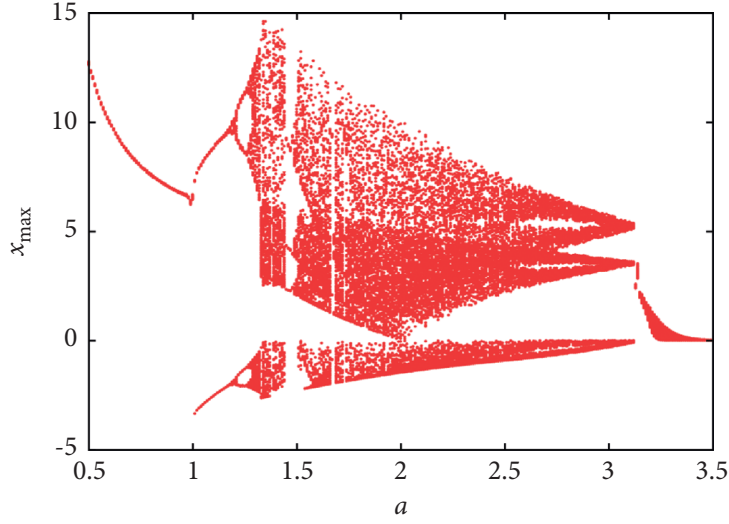

(b)

FiguRE 12: Bifurcation diagrams of the Rucklidge system showing local maxima of the $x$ variable in one complete orbit, $x_{\max }$, as a function of different parameters.

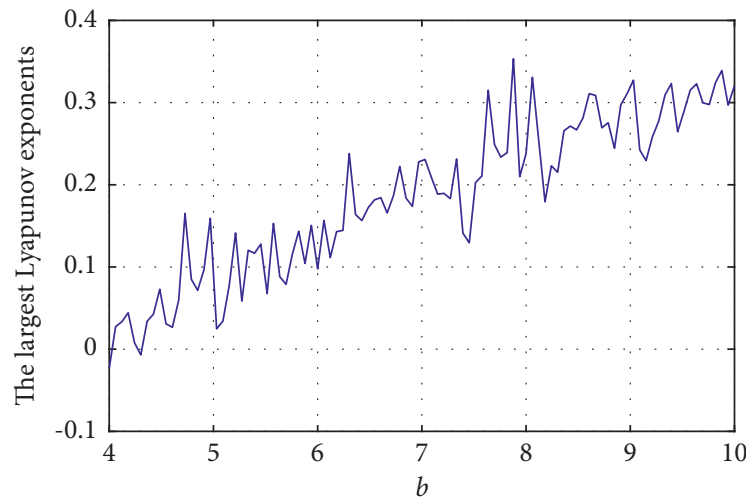

(a)

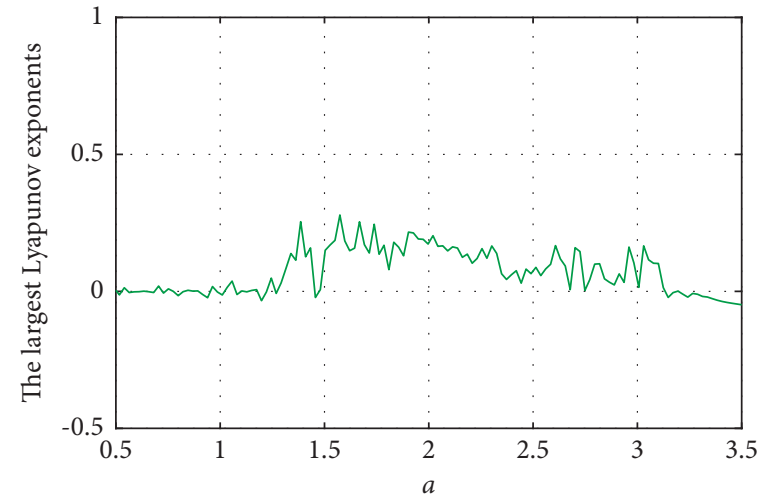

(b)

FIGURE 13: The largest Lyapunov exponents spectrum of the Rucklidge system with respect to different parameters.

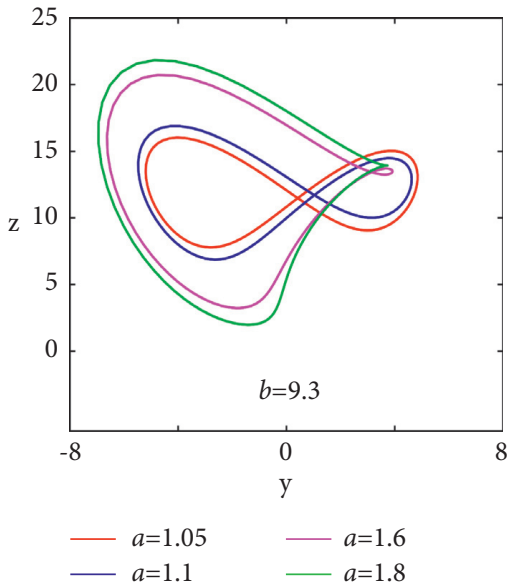

(a)

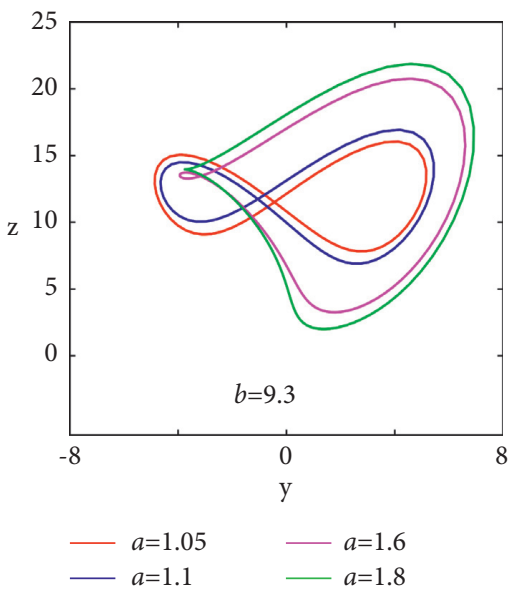

(b)

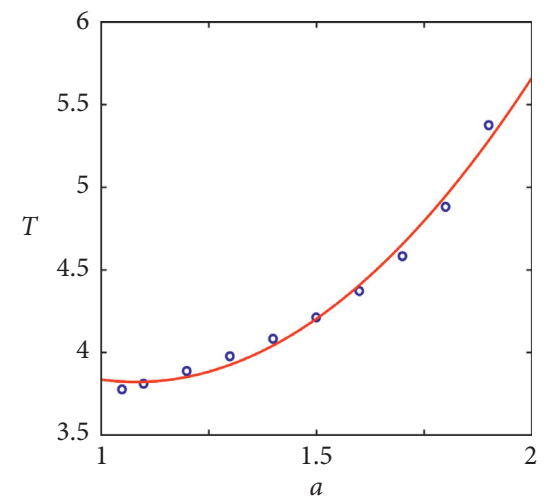

Figure 14: (a, b) Homotopy evolution of cycles 2 and 3 for different $a$ values. (c) Periods of cycle 2 for different $a$ values. A quadratic curve fitting method was adopted to calculate the approximate relationship between them. 


$$
T=2.2 a^{2}-4.7 a+6.3
$$

Tables 3 and 4 show that, for the condition $a>0$, the larger the parameter $a$ is, the larger the period is. In contrast, for $b>0$, as the parameter $b$ increases, the period decreases. We also confirmed that this conclusion is applicable to all the short periodic orbits found.

\section{Conclusions}

In this paper, we studied the topological classification of unstable periodic orbits in the Rucklidge system. Our motivation was to develop an effective method for the symbolic encoding of periodic orbits in the study of nonlinear dynamical systems, enabling us to extend the application of symbolic dynamics to multimodal maps. We demonstrated that symbolic encodings based on trajectory topology are very effective for classifying all short cycles in the Rucklidge system. In addition, upon parameter changes, three different bifurcations, including a pitchfork bifurcation, perioddoubling bifurcation, and saddle-node bifurcation, of the periodic orbits were explored in detail using the homotopy evolution approach, and the evolution rules of the orbital period as parameters varied for all found cycles were also summarized. This provides a new path for charting possible periodic orbits under certain parameters. The symbolic encoding method proposed in this paper is not only suitable for the Rucklidge system but also applicable to other typical chaotic systems. Particularly, it could be extended to solve a large number of concrete physical problems in different regimes, such as recolliding periodic orbits in attosecond physics [49], Newtonian three-body planar periodic orbits [50], and chaos in a hybrid optical bistable system [51].

In some dynamical systems, the unstable periodic orbits are not only embedded in the attractor but also in the nonattracting chaotic set, which is thought to be responsible for chaotic transient dynamics. The unstable periodic orbits outside the attractor, which form chaotic saddles, can also be calculated using the variational method. The topological classification approach employed here seems to be applicable for the symbolic encoding of periodic orbits outside the attractor, thus enabling us to classify the cycles and order them in a hierarchical way. Meanwhile, the construction of symbolic encodings based on orbital topology may also help to label homoclinic and heteroclinic orbits systematically and analyze their associations with each other. Furthermore, the topological structure of an attractor is the direct result of the stretching and folding of the bundles of trajectories simultaneously, whose properties are synthesized by a branched manifold, named template [52-56]. The template describes the topological organization of all the unstable periodic orbits existing in the chaotic attractor, which can be proposed in the manner of the linking matrix using the linking numbers between pairs of cycles extracted from the strange attractor and the Poincaré first return map structure. Hence, the method presented in this paper can be improved to identify the topological structure of chaotic attractors.

The multiple-letter symbolic encoding method may also be applicable to some discrete dynamical systems. How to implement symbolic encoding in dynamical systems with no fixed points remains a challenge. Apparently, there are still rich, intricate characteristics and phenomena of the Rucklidge system in terms of periodic orbits. Therefore, more detailed analyses and explorations will be conducted in future research. We expect that the research in this paper will stimulate further studies on chaos, promoting a deep understanding of the periodic orbits in low-dimensional dissipative chaotic systems.

\section{Data Availability}

The data used to support the findings of this study are available from the corresponding author upon request.

\section{Conflicts of Interest}

The authors declare that there are no conflicts of interest regarding the publication of this study.

\section{Acknowledgments}

This work was supported by the National Natural Science Foundation of China (Grant nos. 11647085 and 11647086), Shanxi Province Science Foundation for Youths (Grant no. 201901D211252), and the Scientific and Technological Innovation Programs of Higher Education Institutions in Shanxi (Grant nos. 2019L0505 and 2019L0554).

\section{References}

[1] E. N. Lorenz, "Deterministic nonperiodic flow," Journal of the Atmospheric Sciences, vol. 20, no. 2, pp. 130-141, 1963.

[2] C. Sparrow, The Lorenz Equations, Bifurcations, Chaos, and Strange Attractors, Springer, Berlin, Germany, 1982.

[3] Q. Lai, Z. Wan, P. D. K. Kuate, and H. Fotsin, "Coexisting attractors, circuit implementation and synchronization control of a new chaotic system evolved from the simplest memristor chaotic circuit," Communications in Nonlinear Science and Numerical Simulation, vol. 89, Article ID 105341, 2020.

[4] X. Wang and G. Chen, "A chaotic system with only one stable equilibrium," Communications in Nonlinear Science and Numerical Simulation, vol. 17, no. 3, pp. 1264-1272, 2012.

[5] A. Saha, "Dynamics of the generalized KP-MEW-Burgers equation with external periodic perturbation," Computers \& Mathematics with Applications, vol. 73, no. 9, pp. 1879-1885, 2017.

[6] O. E. Rössler, "An equation for continuous chaos," Physics Letters A, vol. 57, no. 5, pp. 397-398, 1976.

[7] G. Chen and T. Ueta, "Yet another chaotic attractor," International Journal of Bifurcation and Chaos, vol. 9, no. 7, pp. 1465-1466, 1999.

[8] J. Lü and G. Chen, "A new chaotic attractor coined," International Journal of Bifurcation and Chaos, vol. 12, no. 3, pp. 659-661, 2002.

[9] J. C. Sprott, "Some simple chaotic flows," Physical Review. E, Statistical Physics, Plasmas, Fluids, and Related Interdisciplinary Topics, vol. 50, pp. R647-R650, 1994.

[10] J. C. Sprott, "Some simple chaotic jerk functions," American Journal of Physics, vol. 65, no. 6, pp. 537-543, 1997. 
[11] J. C. Sprott, “A new class of chaotic circuit," Physics Letters A, vol. 266, no. 1, pp. 19-23, 2000.

[12] A. Saha, "Bifurcation, periodic and chaotic motions of the modified equal width-Burgers (MEW-Burgers) equation with external periodic perturbation," Nonlinear Dynamics, vol. 87, no. 4, pp. 2193-2201, 2017.

[13] A. Das and A. Saha, "Dynamical survey of the dual power Zakharov-Kuznetsov-Burgers equation with external periodic perturbation," Computers \& Mathematics with Applications, vol. 76, no. 5, pp. 1174-1183, 2018.

[14] A. M. Rucklidge, "Chaos in models of double convection," Journal of Fluid Mechanics, vol. 237, pp. 209-229, 1992.

[15] Y. Zhang and T. Zhou, "Three schemes to synchronize chaotic fractional-order Rucklidge systems," International Journal of Modern Physics B, vol. 21, no. 12, pp. 2033-2044, 2007.

[16] X. Wang, "Si'lnikov chaos and Hopf bifurcation analysis of Rucklidge system," Chaos, Solitons \& Fractals, vol. 42, no. 4, pp. 2208-2217, 2009.

[17] I. Pehlivan, Y. Uyarolu, and M. Youn, "Chaotic oscillator design and realizations of the Rucklidge attractor and its synchronization and masking simulations," Scientific Research and Essays, vol. 5, pp. 2210-2219, 2010.

[18] F. S. Dias and L. F. Mello, "Hopf bifurcations and small amplitude limit cycles in Rucklidge systems," The Electronic Journal of Differential Equations, vol. 20139 pages, 2013.

[19] S. Nikolov, "First Lyapunov value and bifurcation behavior of specific class of three-dimensional systems," International Journal of Bifurcation and Chaos, vol. 14, no. 8, pp. 2811-2823, 2004.

[20] U. E. Kocamaz and Y. Uyaroğlu, "Controlling Rucklidge chaotic system with a single controller using linear feedback and passive control methods," Nonlinear Dynamics, vol. 75, no. 1-2, pp. 63-72, 2014.

[21] M. Marwan, S. Ahmad, M. Aqeel, and M. Sabir, "Control analysis of Rucklidge chaotic system," Journal of Dynamic Systems, Measurement, and Control, vol. 141, p. 041010, 2018.

[22] D. Zhang, A. Zhao, X. Yang, Y. Sun, and J. Xiao, "Generalized synchronization between Chen system and Rucklidge system," IEEE Access, vol. 7, pp. 8519-8526, 2019.

[23] M. F. S. Lima, J. Llibre, and C. Valls, "Integrability of the Rucklidge system," Nonlinear Dynamics, vol. 77, no. 4, pp. 1441-1453, 2014.

[24] Z. Chen, G. Wen, H. Zhou, and J. Chen, “A new $M \times N$-grid double-scroll chaotic attractors from Rucklidge chaotic system," Optik, vol. 136, pp. 27-35, 2017.

[25] J. F. Gibson, J. Halcrow, and P. Cvitanović, "Equilibrium and travelling-wave solutions of plane Couette flow," Journal of Fluid Mechanics, vol. 638, pp. 243-266, 2009.

[26] S. H. Strogatz, Nonlinear Dynamics and Chaos: With Applications to Physics, Biology, Chemistry, and Engineering, Perseus Books Publishing, Cambridge, UK, 2000.

[27] R. Artuso, E. Aurell, and P. Cvitanovic, "Recycling of strange sets: I. cycle expansions," Nonlinearity, vol. 3, no. 2, pp. 325-359, 1990.

[28] R. Artuso, E. Aurell, and P. Cvitanovic, "Recycling of strange sets: II. applications," Nonlinearity, vol. 3, no. 2, pp. 361-386, 1990.

[29] C. Dong, "Topological classification of periodic orbits in the Yang-Chen system," EPL (Europhysics Letters), vol. 123, no. 2, Article ID 20005, 2018.

[30] Y. Lan, "Cycle expansions: from maps to turbulence," Communications in Nonlinear Science and Numerical Simulation, vol. 15, no. 3, pp. 502-526, 2010.
[31] P. Cvitanović, R. Artuso, R. Mainieri, G. Tanner, and G. Vattay, Chaos: Classical and Quantum, Niels Bohr Institute, Copenhagen, Denmark, 2012.

[32] Y. Lan and P. Cvitanović, "Variational method for finding periodic orbits in a general flow," Physical review. E, Statistical, nonlinear, and soft matter physics, vol. 69, Article ID 016217, 2004.

[33] B.-L. Hao and W.-M. Zheng, Applied Symbolic Dynamics and Chaos, World Scientific, Singapore, 1998.

[34] S. D. Ruzza, J. Daquin, and G. Pinzari, "Symbolic dynamics in a binary asteroid system," Communications in Nonlinear Science and Numerical Simulation, vol. 91, Article ID 105414, 2020.

[35] D. Arroyo and G. Alvarez, "Application of gray codes to the study of the theory of symbolic dynamics of unimodal maps," Communications in Nonlinear Science and Numerical Simulation, vol. 19, no. 7, pp. 2345-2353, 2014.

[36] Y. Lan and P. Cvitanović, "Unstable recurrent patterns in Kuramoto-Sivashinsky dynamics," Physical Review E-Statistical Physics, Plasmas, Fluids, and Related Interdisciplinary Topics, vol. 78, Article ID 026208, 2008.

[37] C. Dong, "Topological classification of periodic orbits in Lorenz system," Chinese Physics B, vol. 27, Article ID 080501, 2018.

[38] C. Dong, P. Wang, M. Du, T. Uzer, and Y. Lan, "The ionized electron return phenomenon of Rydberg atom in crossedfields," Modern Physics Letters B, vol. 30, Article ID 1650183, 2016.

[39] P. Cvitanović, Universality in Chaos, Adam Hilger, Bristol, England, 2nd edition, 1989.

[40] W. H. Press, S. A. Teukolsky, W. T. Veterling, and B. P. Flannery, Numerical Recipes in C, Cambridge University Press, Cambridge, UK, 1992.

[41] Y. Lan, C. Chandre, and P. Cvitanović, "Newton's descent method for the determination of invariant tori," Physical review. E, Statistical, nonlinear, and soft matter physics, vol. 74, Article ID 046206, 2006.

[42] C. Dong and Y. Lan, "A variational approach to connecting orbits in nonlinear dynamical systems," Physics Letters A, vol. 378, no. 9, pp. 705-712, 2014.

[43] C. Dong, H. Liu, and H. Li, "Unstable periodic orbits analysis in the generalized Lorenz-type system," Journal of Statistical Mechanics: Theory and Experiment, vol. 2020, Article ID 073211, 2020.

[44] C. Dong and Y. Lan, "Organization of spatially periodic solutions of the steady Kuramoto-Sivashinsky equation," Communications in Nonlinear Science and Numerical Simulation, vol. 19, no. 6, pp. 2140-2153, 2014.

[45] A. Ray, D. Ghosh, and A. R. Chowdhury, "Topological study of multiple coexisting attractors in a nonlinear system," Journal of Physics A: Mathematical and Theoretical, vol. 42, Article ID 385102, 2009.

[46] M. Tarnowski, F. N. Ünal, N. Fläschner et al., "Measuring topology from dynamics by obtaining the Chern number from a linking number," Nature Communications, vol. 10, p. 1728, 2019.

[47] R. Ahmad, S. Paul, and S. Basu, "Characterization of entanglements in glassy polymeric ensembles using the Gaussian linking number," Physical Review, vol. 101, Article ID 022503, 2020.

[48] A. A. Didov, M. Y. Uleysky, and M. V. Budyansky, "Stable and unstable periodic orbits and their bifurcations in the nonlinear dynamical system with a fixed point vortex in a periodic 
flow," Communications in Nonlinear Science and Numerical Simulation, vol. 91, Article ID 105426, 2020.

[49] A. Kamor, F. Mauger, C. Chandre, and T. Uzer, "How key periodic orbits drive recollisions in a circularly polarized laser field," Physical Review Letters, vol. 110, Article ID 253002, 2013.

[50] M. Šuvakov and V. Dmitrašinović, "Three classes of Newtonian three-body planar periodic orbits," Physical Review Letters, vol. 110, Article ID 114301, 2013.

[51] M.-S. Abdelouahab and N.-E. Hamri, "A new chaotic attractor from hybrid optical bistable system," Nonlinear Dynamics, vol. 67, no. 1, pp. 457-463, 2012.

[52] C. Letellier, P. Dutertre, and B. Maheu, "Unstable periodic orbits and templates of the Rössler system: toward a systematic topological characterization," Chaos: An Interdisciplinary Journal of Nonlinear Science, vol. 5, no. 1, pp. 271-282, 1995.

[53] T. D. Tsankov and R. Gilmore, "Topological aspects of the structure of chaotic attractors in $R^{3}$," Physical Review E-Statistical Physics, Plasmas, Fluids, and Related Interdisciplinary Topics, vol. 69, Article ID 056206, 2004.

[54] T. D. Tsankov and R. Gilmore, "Strange attractors are classified by bounding tori," Physical Review Letters, vol. 91, Article ID 134104, 2003.

[55] M. Rosalie, "Templates and subtemplates of Rössler attractors from a bifurcation diagram," Journal of Physics A: Mathematical and Theoretical, vol. 49, Article ID 315101, 2016.

[56] M. Rosalie and C. Letellier, "Toward a general procedure for extracting templates from chaotic attractors bounded by high genus torus," International Journal of Bifurcation and Chaos, vol. 24, Article ID 1450045, 2014. 